\title{
MOLECULAR OUTFLOWS
}

\author{
Ronald L. Snell \\ Five College Radio Astronomy Observatory and the Department \\ of Physics and Astronomy, University of Massachusetts, Amherst
}

\section{Introduction}

The development of millimeter and submillimeter spectroscopy has allowed astronomers to probe the cold, dense component of the interstellar medium. This medium, primarily composed of gas in molecular form, is gravitationally bound into relatively distinct clouds that are sites of star formation within our Galaxy. The most ubiquitous tracers of these molecular clouds are the rotational transitions of carbon monoxide. Observations of $\mathrm{CO}$ emission have been commonly used to estimate the size, temperature, mass, and density of molecular clouds; in addition, the spectral line profiles can be used to study the internal dynamics of these clouds. Although the sound speed within molecular clouds is only about $0.2 \mathrm{~km} \mathrm{~s}^{-1}$, observed CO line widths are more typically 1 to $5 \mathrm{~km} \mathrm{~s}^{-1}$. Thus, the internal dynamics of molecular clouds are characterized by supersonic gas motions whose nature is poorly understood.

In addition to the generally supersonic gas motions present in molecular clouds, there are localized regions of much faster hypersonic gas motions associated with newly forming stars. These motions are understood to represent expanding gas flows from young stellar objects. An example of hypersonic molecular flow is illustrated in Figure 1a, in which a series of CO spectra obtained along a line from northwest to southeast through the infrared source associated with HH7-11 are displayed (Snell and Edwards 1981). The line widths away from the position of the infrared source ( $3 \mathrm{~N}-3 \mathrm{~W}$ and $2 \mathrm{~S}-2 \mathrm{E}$ ) are dominated by the supersonic gas motions in the cloud, but towards the infrared source the total velocity extent of the emission is much greater, arising from gas outflowing from the young star embedded within the molecular cloud.

One of the most interesting aspects of molecular outflows is their bipolar nature (Snell, Loren, and Plambeck 1980). This can be seen in the maps of the redshifted and blueshifted emissions presented in Figure 1b for the HH7-11 region; the redshifted emission extends to the northwest while the blueshifted emission extends to the southeast. Presumably, the winds from the young star at the center of $\mathrm{HH} 7-11$ are collimated into two oppositely directed jets that accelerate the surrounding molecular material. This produces the bipolar signature seen in the high velocity $\mathrm{CO}$ emission. Further evidence for highly collimated stellar winds is also provided by detection of optical jets (see Mundt 1988; Strom et al. 1986). 

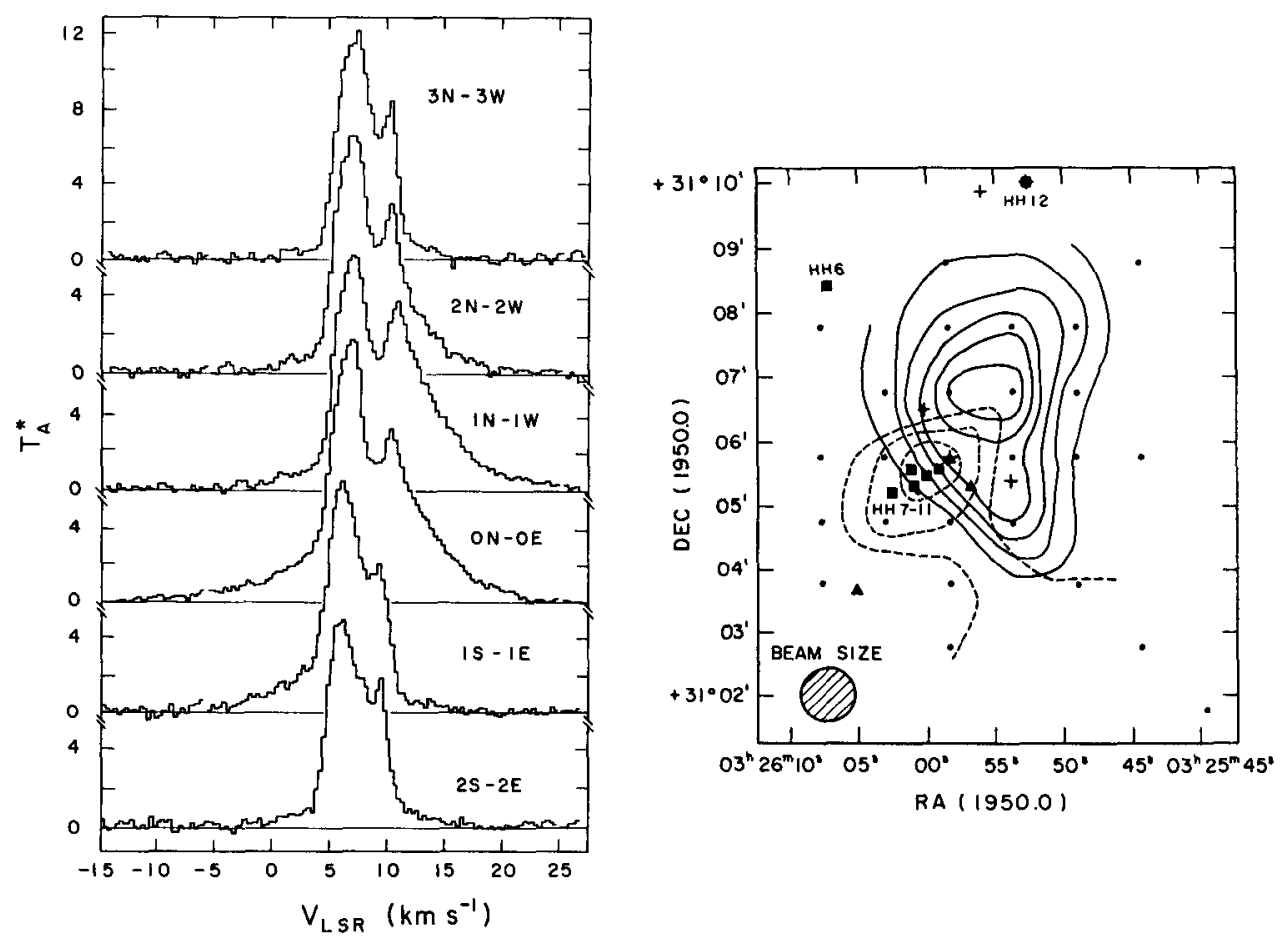

Figure 1a (left). - CO $\mathrm{J}=2-1$ spectra obtained at six locations along a southeast to northwest axis through the infrared source associated with $\mathrm{HH} 7-11$. The offset position of each spectrum in arcminutes north, south, east, and west of the IR sources are indicated.

Figure 1b (right). - A map of the integrated intensity of the high velocity redshifted (solid contours) and blueshifted (dashed contours) $\mathrm{CO}$ emissions in the $\mathrm{HH} 7-11$ region. Both figures are from Snell and Edwards (1981).

A vast body of data has been collected over the past decade which addresses the physical properties and nature of molecular outflows. Only a brief summary of this literature will be presented, since it is thoroughly covered in the recent reviews by Lada (1985) and Snell (1987). Instead, most of this review will be devoted to a summary of the more recent developments in this field.

\section{Summary of Molecular Outflow Properties}

The properties of molecular outflows have been summarized by Lada (1985) and Snell (1987), and I refer readers to these reviews for a more detailed description of the properties of molecular outflows. Briefly, molecular outflows have hypersonic gas motions with velocities of 10 to $100 \mathrm{~km} \mathrm{~s}^{-1}$ and are associated with young stellar objects (YSOs). The mass of the 
hypersonic molecular gas $\left(0.1\right.$ to $\left.100 \mathrm{M}_{\odot}\right)$ contains kinetic energies of $10^{43}$ to $10^{47} \mathrm{ergs}$. The outflow phenomenon is short lived with dynamical timescales $\leq$ few $\mathrm{x} 10^{5}$ years; thus, massive stellar winds (with mass loss rates of $10^{-7}$ to $10^{-3} \mathrm{M}_{\odot} \mathrm{yr}^{-1}$ for wind velocities of $200 \mathrm{~km} \mathrm{~s}^{-1}$ ) are required. Approximately $75 \%$ of all known outflows show bipolar morphologies, suggesting that the winds from young stars in general are collimated. Outflows are associated with both high and low luminosity young stars $\left(1\right.$ to $\left.10^{5} \mathrm{~L}_{\odot}\right)$ and the energies and momenta in the outflows are correlated with the radiant luminosity. Typical properties of an outflow are summarized in Table 1. Finally, a large number of outflows have been detected, suggesting that the outflows represent a common evolutionary phase of many young stars.

TABLE 1

Typical Molecular Outflow Properties

\begin{tabular}{ll}
\hline \hline Mass & $3 \mathrm{M}_{\odot}$ \\
Velocity extent & $25 \mathrm{~km} \mathrm{~s}^{-1}$ \\
Size & $0.5 \mathrm{pc}$ \\
Energy & $10^{45} \mathrm{ergs}$ \\
Momentum & $20 \mathrm{M}_{\odot} \mathrm{km} \mathrm{s}^{-1}$ \\
Dynamical timescale & $5 \times 10^{4} \mathrm{years}^{-1} \mathrm{Mr}^{-1}$ \\
Force & $10^{-3} \mathrm{M}_{\odot} \mathrm{km} \mathrm{s}^{-1} \mathrm{yr}^{-1}$ \\
Mass loss rate & $5 \times 10^{-6} \mathrm{M}_{\odot} \mathrm{yr}^{-1}$ \\
\hline
\end{tabular}

The data on molecular outflows summarized in the reviews of Lada (1985) and Snell (1987) are extensive, but still a number of fundamental questions concerning the nature of these outflows remain to be answered. Some of these are:

- What is the evolutionary status of the YSOs responsible for molecular outflows?

- What is the frequency of outflow events and what type (mass) stars produce outflows?

- What is the structure and kinematics of the outflowing molecular gas?

- What impact do outflows have on stellar evolution and on the evolution of the surrounding molecular cloud?

- What is the nature of the stellar wind that drives the molecular outflows?

- How are these stellar winds generated and collimated?

A summary of recent studies that have attempted to answer many of these questions will be presented in the following sections. Three topics will be emphasized: 1.) outflow surveys, 2.) the structure of molecular outflows, and 3.) the nature of the stellar winds. 


\section{Outflow Surveys}

Past searches for outflows were neither systematic nor statistially complete, owing primarily to the lack of a good census of YSOs in molecular clouds. While past surveys either relied on optical catalogs, or incomplete infrared surveys to identify YSOs, the data available from the Infrared Astronomical Satellite (IRAS) have now allowed for a more complete census. In addition, totally unbiased searches for outflows have also been undertaken in a number of molecular clouds; these allow for the detection of outflows associated with YSOs below the IRAS detection threshold or in confused regions of the sky in the far-infrared. Together, such surveys have provided a more definitive determination of the frequency of energetic outflows and the evolutionary status of the young stars responsible for them.

\section{a.) Outflows Associated with Luminous FIR Sources}

A flux limited sample of IRAS sources has been studied by Snell et al. (1988) and Snell Dickman, and Huang (1989). Their sample consisted of all $100 \mu \mathrm{m}$ sources with flux densities $>$ $500 \mathrm{Jy}$ found in the range $0^{\mathrm{h}}<\alpha<12^{\mathrm{h}}$ and $\delta>0^{\circ}$. Based on both their far-infrared colors and their close association with strong $\mathrm{CO}$ emission, all members of this sample are believed to be relatively luminous $\left(10^{4} \mathrm{~L}_{\odot}\right)$ YSOs still deeply embedded in the clouds from which they formed. Among the 51 sources in the sample, 12 were already known to have associated molecular outflows (Lada 1985). The 39 remaining sources were surveyed and 11 new outflows detected. Thus, the detection rate of outflows associated with luminous YSOs is nearly $50 \%$, similar to detection rate of outflow candidates by Casoli et al. (1986).

The high incidence of outflows in these surveys can be used to set an upper limit on the lifetime of objects as bright far-infrared sources. If these sources lived considerably longer than the duration of the outflow phase, then one would expect to have a very low rate of outflow detection and based on the $50 \%$ detection rate, the maximum lifetime of a FIR source is roughly twice the outflow lifetime. The average age of the 11 outflows studied is estimated to be $2 \times 10^{5}$ years; therefore, the material surrounding these young $O$ and $B$ stars must be dispersed within about $4 \times 10^{5}$ years. The cloud dispersal and outflow phenomena must be concurrent and begin shortly after a young star begins to generate any substantial luminosity.

\section{b.) Far-infrared Sources with $L<100 \mathrm{~L}_{\odot}$}

A number of searches have also been undertaken to identify outflows associated with low luminosity far-infrared sources. A flux limited sample of IRAS sources in the Taurus Dark Clouds was surveyed for outflows by Heyer et al. (1987). Their detection rate of $3 / 30$ (outflows detected/sources observed) was relatively low, but most of the sources observed were optically visible stars. In fact, their detection rate was similar to an earlier outflow survey of an optical 
sample of T Tauri stars (Edwards and Snell 1982). Since low luminosity, and presumably low mass, stars have long pre-main sequence lifetimes and because the outflow phenomenon is short lived, it is not surprising that the detection rate is very low.

Studies of outflows associated with IRAS sources within $\mathrm{NH}_{3}$ cores have been completed by Myers et al. (1988) and Terebey, Vogel, and Myers (1989). These sources are thought to be the youngest of the YSOs because they are still associated with the dense gas out of which they were formed. For IRAS sources embedded within $\mathrm{NH}_{3}$ cores they found an outflow detection rate of $16 / 30$. Five additional sources showed broad line widths, but were not mapped to confirm their outflow nature; thus, as many as 21/30 IRAS sources in cores could be associated with outflows. On the other hand, for IRAS sources outside of cores the detection rate was $0 / 8$, and for cores without stars the detection rate was $0 / 4$. Thus, outflow activity is apparently associated with only the youngest infrared sources. Based again on the outflows' short duration, the outflow phenomenon must begin early in the evolution of young, low mass stars, probably within the first $10^{5}$ years. Myers et al. (1988) also found that outflows have sufficient energy, duration, and frequency to be the main agent of core dispersal in molecular clouds. Thus, after the outflows have removed the surrounding dense and visually opaque material, the stars still have long pre-main sequence lifetimes as optically visible T Tauri stars.

\section{c.) Unbiased Surveys}

It is necessary to have a complete census of outflows within a molecular cloud in order to assess their impact on cloud evolution. Since the IRAS Point Source Catalog may be incomplete and since outflow activity may begin before appreciable radiant energy is generated, totally unbiased surveys may be needed to accurately obtain a census of outflows. The first survey of this kind was made by Margulis and Lada (1986) in the Mon OB1 molecular cloud. They surveyed roughly 1.5 square degrees encompassing a cloud mass of $3 \times 10^{4} \mathrm{M}_{\odot}$. Additional studies by Margulis, Lada, and Snell (1988) and Margulis, Lada, and Young (1989) provided detailed maps of the outflows and attempted to identify the YSOs responsible. Nine molecular outflows were detected, associated with the youngest and most luminous IRAS sources within the cloud. The energy and momentum injected into the molecular cloud are also apparently sufficient to maintain the turbulent support of the clouds against gravity, even if the turbulent energy is dissipated on a free-fall timescale.

One of the most energetic outflows identified in the Mon OB1 survey, that labeled G, does not have any obvious optical or IRAS emission associated with it and, thus, must have a relatively low bolometric luminosity. This emphasizes that unbiased surveys may be essential in order to obtain a complete inventory of outflows within molecular clouds. 
A more ambitious outflow search by Fukui and collaborators (Fukui et al. 1986; Fukui 1989 ) is in progress on the Nogoya $4 \mathrm{~m}$ telescope. They have completed an unbiased survey of four clouds (Orion N, Orion S, Mon R2, and S287) and have begun similar surveys in a number of other clouds. They have detected 50 new outflows, 41 of which lie within $1 \mathrm{kpc}$ of the sun. In the clouds that have been completely sampled, outflows are found associated with the coldest (based on 12 and $25 \mu \mathrm{m}$ flux densities) and most luminous YSOs in these clouds. In the Orion S cloud a total of 12 outflows have been identified and these may well contribute substantially to the turbulent support of this cloud.

\section{d.) Summary}

The number of known outflows is increasing steadily as more molecular clouds in the solar vicinity are surveyed. The number of outflows known to lie within $1 \mathrm{kpc}$ of the sun is $>100$; their average lifetime is approximately $3 \times 10^{4}$ years. Thus, the formation rate of outflow progenitor stars is $>10^{-3} \mathrm{yr}^{-1} \mathrm{kpc}^{-2}$. Comparing this rate of formation to that of field stars in the solar neighborhood (Miller and Scalo 1979), implies that all stars greater than $1 \mathrm{M}_{\odot}$ must produce detectable outflows in their lifetime. This not only indicates that both high and low mass stars produce molecular outflows, but that basically all stars must pass through an evolutionary phase in which they produce energetic winds. This phase for both high and low mass stars must occur within about $10^{5}$ years after a source begins generating any substantial luminosity. In addition, outflows may be the primary agent to disperse the dense gas surrounding these stars. Finally, the momentum carried by the dispersed gas may play an important role in maintaining the turbulent support of clouds; this turbulence may be the origin of the supersonic gas motions in clouds.

\section{Structure of Molecular Outflows}

The structure and kinematics of outflowing molecular gas provide important clues to its energetics and origin. A summary of recent observations of three molecular outflows, L1551, B335, and NGC2071 is presented below.

L1551 is a nearby molecular cloud with a well collimated outflow of large angular size (30'). Recent studies by Moriarty-Schieven et al. (1987), Uchida et al. (1987), Rainey et al. (1987), Moriarty-Schieven and Snell (1988), and Fridlund et al. (1989) have provided high resolution images of the $\mathrm{CO}$ emission. One of the most important conclusions reached by these investigators, primarily from the limb-brightened appearence of the molecular emission, is that the high velocity molecular emission arises from an expanding shell of material. The high velocity blueshifted and redshifted emissions are shown in Figure 2. The blueshifted lobe shows clearly that the largest column density of high velocity gas is found at the periphery of the 


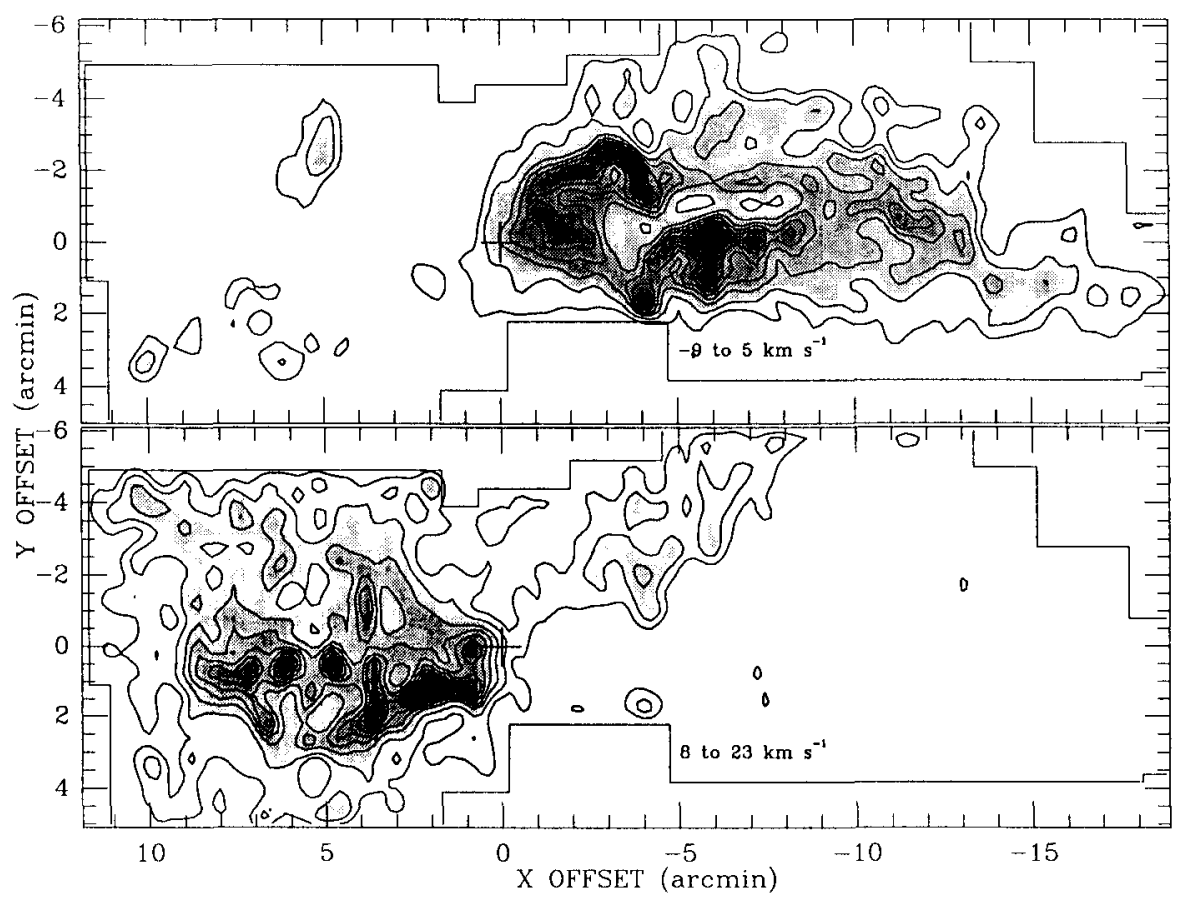

Figure 2. - A map of the high velocity blueshifted (upper) and redshifted (lower) CO emissions in the L1551 outflow obtained by Moriarty-Schieven and Snell (1988). The $\mathrm{x}$-axis is parallel to the outflow axis (at a position angle of $45^{\circ}$, see Figure 2) and the origin of the $x-y$ coordinate system is the position of L1551 IRS-5 (maked by a cross).

outflow. Further evidence for a shell structure is provided by the detection of optical reflection nebulosity coincident with the regions of high column density in the blueshifted lobe. Presumably, light from IRS-5 is able to propagate across the nearly evacuated cavity created by the stellar wind and reflect off the walls of swept-up molecular gas. Expansion of this shell perpendicular to the outflow axis can be seen in the spatial-velocity diagram shown in Figure 3 . The diagram shows the ambient cloud emission at $6.5 \mathrm{~km} \mathrm{~s}^{-1}$ as well as the high velocity blueshifted emission; the circular pattern is indicative of expansion with a velocity of about $3 \mathrm{~km}$ $\mathrm{s}^{-1}$. The total mass in the molecular outflow is estimated to be $3.5 \mathrm{M}_{\odot}$. Both the large mass and the morphology argue strongly for the high velocity molecular gas to be primarily accelerated ambient cloud material.

Extended far-infrared emission has also been detected from the L1551 outflow (Clark and Laureijs 1986; Edwards et al. 1986). The total luminosity is estimated to be $7 \mathrm{~L}_{\odot}, 35$ times larger than the mechanical luminosity in the molecular outflow, but still significantly less than the bolometric luminosity of IRS-5. The extended infrared emission is unlikely to be due to 


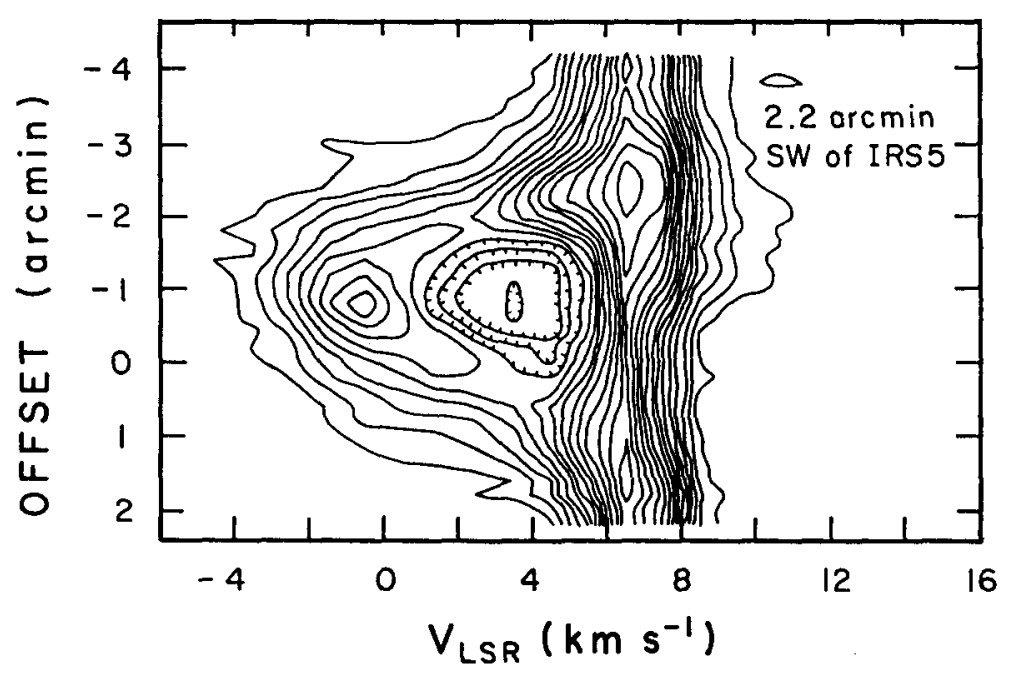

Figure 3. - A spatial-velocity diagram of the CO emission in the blueshifted lobe of the L1551 outflow (Moriarty-Schieven et al. 1987). The spatial axis is oriented perpendicular to the outflow axis and located 2.2' (X offset $=-2.2$ ) from IRS-5.

dust heated by stellar photons and probably arises from the stellar wind from IRS-5. If most of the stellar wind energy is radiated away at the wind shock located at the boundary with the swept-up molecular shell, this could account for the observed far-infrared luminosity.

The molecular outflow in B335 is also relatively nearby $(250 \mathrm{pc})$. Several recent papers (Cabrit, Goldsmith, and Snell 1988; Hirano et al. 1988; Moriarty-Schieven and Snell 1989) have presented high resolution images of the high velocity $\mathrm{CO}$ emission. The data show that, like L1551, the high velocity emission appears to be confined to a shell. One of the most peculiar aspects of the B335 outflow is the multiple patterns of superimposed blueshifted and redshifted emissions. These patterns have been attributed to the presence of two independent outflows, but the new data demonstrate that B335 is in fact a single, highly collimated outflow, oriented nearly in the plane of the sky. The superimposed blueshifted and redshifted emissions arise from the front and back sides of the expanding shell. The angle of inclination out of the plane of the sky is estimated to be between 8 and $10^{\circ}$. Thus, the B335 outflow is very similar to that in L1551, except viewed at a smaller inclination angle.

NGC2071 lies at a distance of $390 \mathrm{pc}$ and like L1551 and B335, its outflow shows a very collimated structure. Moriarty-Schieven, Snell, and Hughes (1989) have recently obtained a high resolution map of $\mathrm{CO}$ emission from the $\mathrm{J}=2-1$ transition. The outflow as a shell morphology similar to L1551, but is much more inclined out of the plane of the sky. The similarity to the L1551 outflow is quite striking and many of the observed differences between the two outflows can be attributed to the larger inclination angle at which the NGC2071 outflow is viewed. 
Generally, high velocity molecular emission appears to arise primarily from an expanding shell driven by a stellar wind. Many of the observed differences in molecular outflows can be attributed to the inclination angle at which they are viewed. Small inclination angle outflows (such as B335 and L1551) have low velocity emission only, are highly collimated, have little overlap of their outflow lobes, and have a readily detectable expansion component. Intermediate inclination angle outflows (such as NGC2071 and $\mathrm{HH} 7-11$ ) have moderate velocity emission, are bipolar but not well collimated, have lobes that overlap, and have their observed kinematics dominated by their axial motion. Large inclination angle outflows (possibly AFGL490) have high velocity emission, are poorly collimated, have total overlap of their lobes, and have their expansion directed perpendicular to line of sight.

\section{The Stellar Wind}

The basic morphology of molecular outflows is illustrated in Figure 5. The most plausible origin of the high velocity molecular gas is ambient cloud material accelerated by a much faster

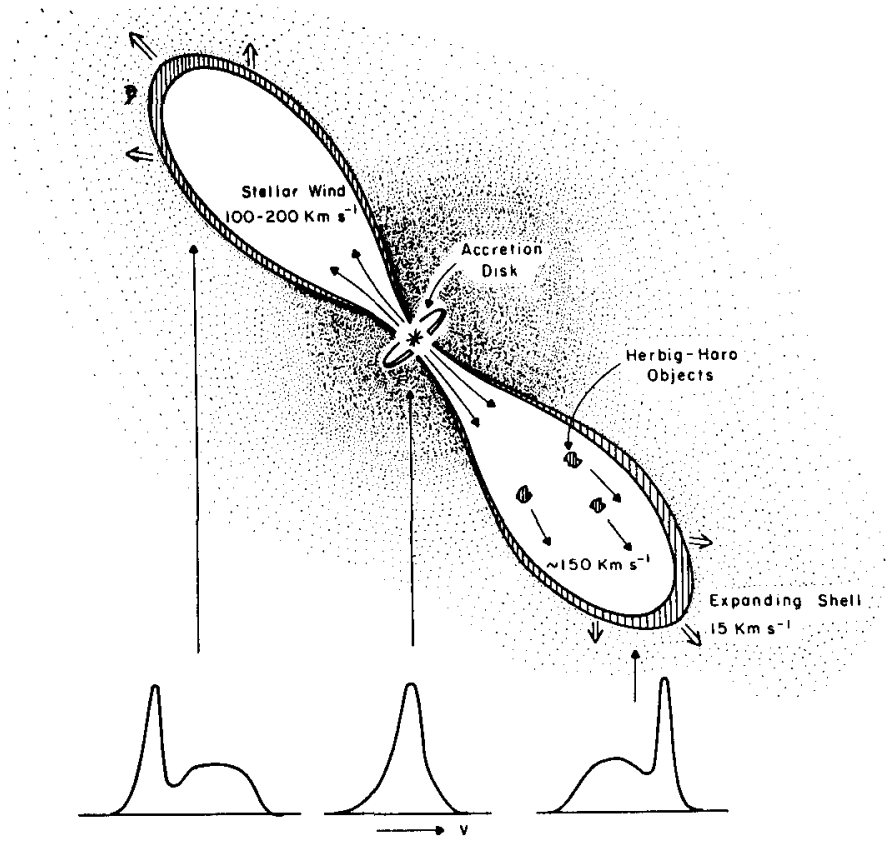

Figure 5 - A schematic diagram showing the morphology of the stellar wind driven molecular shell and the location of the shock-excited Herbig-Haro objects. 
stellar wind. What is the nature of this stellar wind ? Rodriguez and Canto (1983), Snell et al. (1985), and Snell and Bally (1986) have searched for the radio continuum emission that would be produced if the stellar winds were ionized. In the cases where free-free emission was detected the mass loss rate of ionized gas was not sufficient to drive the molecular outflows. Levreault (1985) and Strom et al. (1986) found similar results in their studies of optical jets. Thus, although collimated jets are seen at radio and optical wavelengths, the ionized gas responsible for the emission must represent a small fraction of the total mass present in the stellar wind.

Recently, extremely high-velocity atomic hydrogen emission has been detected from HH7-11 by Lizano et al. (1988). The emission implies wind velocities as large as $170 \mathrm{~km} \mathrm{~s}^{-1}$ and indicates a mass loss rate of $3 \times 10^{-6} \mathrm{M}_{\odot} \mathrm{yr}^{-1}$, sufficient to drive the associated molecular outflow. Thus, in this one outflow the stellar wind is clearly neutral and atomic. In L1551, the HI observations made by Lizano et al. are more ambiguous but there is also evidence for high velocity $\mathrm{HI}$ emission that suggests the possibility for a neutral stellar wind with a line of sight velocity of $50 \mathrm{~km} \mathrm{~s}^{-1}\left(190 \mathrm{~km} \mathrm{~s}^{-1}\right.$ after applying an inclination correction). The mass loss rate in this stellar wind may also be sufficient to drive the more massive molecular outflow and account for the excess far-infrared emission seen in this source.

An equally remarkable discovery made by Lizano et al. was the detection of extremely high velocity molecular emission in the HH7-11 outflow. The CO emission has a velocity extent of $\pm 160 \mathrm{~km} \mathrm{~s}^{-1}$, considerably larger than the velocities seen in Figure 1b. Lizano et al. suggested that the CO emission arises from molecular gas entrained in a neutral stellar wind. Alternatively, chemical modeling of neutral winds (Glassgold, Mamon, and Huggins 1988) suggests that it is possible to form an appreciable amount of $\mathrm{CO}$ from the atomic gas. The abundance of $\mathrm{CO}$ relative to $\mathrm{H}$ in the stellar wind is strongly dependent on the mass loss rate of the driving source, but for large mass loss rates $\left(>10^{-5} \mathrm{M}_{\odot} \mathrm{yr}^{-1}\right)$ the abundance of $\mathrm{CO}$ approaches that found in the ambient molecular material.

Observations to detect extremely high velocity $\mathrm{CO}$ emission from stellar winds have been carried out by Koo (1989) and by Margulis and Snell (1989). They detected weak, but extremely high velocity, emission in a number of sources known to have molecular outflows. An example is shown in Figure 5 for NGC2071 where emission is detected over a velocity range $>110 \mathrm{~km} \mathrm{~s}^{-1}$. In fact, the high velocity emission appears to be readily separated into contributions from the molecular shell and the stellar wind. The break in the power law shape of the line wings, indicated in Figure 5, may indicate the division of the gas into these two components; emission at temperatures below about $0.1 \mathrm{~K}$ may arise primarily from molecular gas in a stellar wind. Extremely high velocity emission is detected only in those outflows with mass loss rates in excess of $10^{-6} \mathrm{M}_{\odot} \mathrm{yr}^{-1}$. Thus, these observations suggest that significant stellar winds exist and that these winds are primarily neutral and atomic. Such winds may be sufficient to drive even the more massive known molecular outflows. 


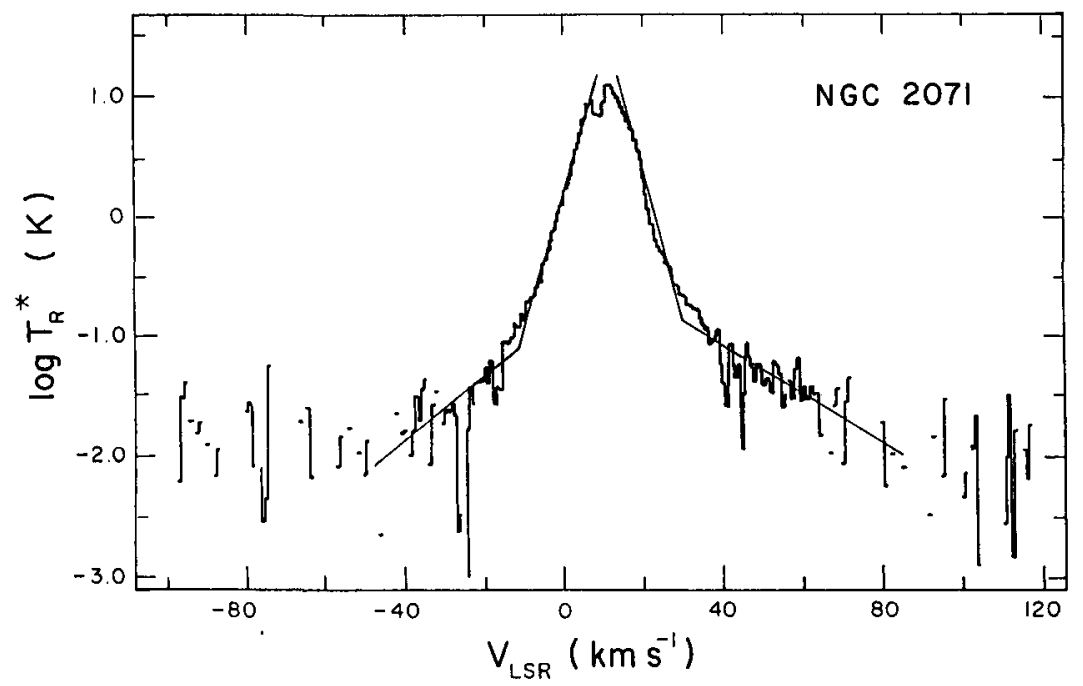

Figure 5. - Logarithm of the spectrum of NGC2071 from Margulis and Snell (1989). Data points in which the antenna temperature dropped below the baseline are not plotted.

\section{Conclusions}

Outflows are evidently a common evolutionary phase for stars of all masses. The outflow phenomenon occurs within the first $10^{5}$ years of a YSO's life and is likely responsible for dispersing the dense gas surrounding it. The bulk of the high velocity molecular emission in an outflow arises from ambient cloud material swept up into an expanding shell by a faster, and more energetic stellar wind. This wind is largely neutral, atomic, and highly collimated into opposed jets. Finally, the energy and momentum carried away by the wind may have important consequences for both the star formation process and for the turbulent support of molecular clouds against gravity.

\section{References}

Cabrit, S., Goldsmith, P.F., and Snell, R.L. 1988, Ap. J., 334, 196.

Casoli, F., Dupraz, C., Gerin, M., Combes, F., and Boulanger, F. 1986, Astron. Astrophy., 169, 281.

Clark, F.O., and Laureijs, R.J. 1986, Astron. Astrophys., 154, L26.

Edwards, S., and Snell, R.L. 1982, Ap. J., 261, 151.

Edwards, S., Strom, S.E., Snell, R.L., Jarrett, T.H., Beichman, C.A., and Strom, K.M. 1986, Ap. $J$. (Letters), 307, L65.

Fridlund, C.V.M., Sandqvist, Aa., Nordh, H.L., and Olofsson, G. 1989, Astron. Astrophys., 213, 310.

Fukui, Y. 1989, private communication. 
Fukui, Y., Sugitani, H., Takaba, H., Iwata, T., Mizuno, A., Ogawa, H., and Kawabata, K 1986, Ap. J. (Letters), 311, L85.

Glassgold, A.E., Mamon, G.A., and Huggins, P.J. 1989, Ap. J. (Letters), 336, L29.

Heyer, M.H., Snell, R.L., Goldsmith, P.F., and Myers, P.C. 1987, $A p$. J., 321, 370.

Hirano, N., Kameya, O., Nakayama, M., and Takakubo, K. 1988, Ap. J. (Letters), 327, L69.

Koo, B.-C. 1989, Ap. J., 337, 318.

Lada, C.J. 1985, Ann. Rev. Astron. Astrophys., 23, 267.

Levreault, R.M. 1985, Ph.D. thesis, University of Texas.

Lizano, S., Heiles, C., Rodriguez, L.F., Koo, B.-C., Shu, F.H., Hasegawa, T., Hayashi, S., and Mirabel, I.F. $1988, A p$. J., 328, 763 .

Margulis, M., and Lada, C.J. 1986, Ap. J. (Letters), 309, L87.

Margulis, M., Lada, C.J., and Snell, R.L. 1988, Ap. J., 333, 316.

Margulis, M., Lada, C.J., and Young, E.T. 1989, preprint.

Margulis, M., and Snell, R.L. 1989, Ap. J., in press.

Miller, G.E., and Scalo, J.M. 1979, Ap. J. Suppl., 41, 513.

Moriarty-Schieven, G.H., Snell, R.L., Strom, S.E., Schloerb, F.P., Strom, K.M., and Grasdalen, G.L. 1987, $A p$. J., 319, 742.

Moriarty-Schieven, G.H., and Snell, R.L. 1988, Ap. J., 332, 364.

Moriarty-Schieven, G.H., and Snell, R.L. 1989, Ap. J., 338, 952.

Moriarty-Schieven, G.H., Snell, R.L., and Hughes, V. 1989, Ap. J., in press.

Mundt, R. 1988, in NATO-ASI on Formation and Evolution of Low Mass Stars, eds. A. Dupree and M.T.V. Lago (Dordrecht: Reidel).

Myers, P.C., Heyer, M., Snell, R.L., and Goldsmith, P.F. 1988, Ap. J., 324, 907.

Rainey, R., White, G.J., Richaredson, K.J., Griffen, M.J., Cronin, N.J., Monterio, T.S., and Hilton, J. 1987, Astro. Astrophys., 179, 237.

Rodriguez, L.F., and Canto, J. 1983, Rev. Mexicana Astr. Ap., 8, 163.

Snell, R.L. 1987, in IAU Symposium 115, Star Forming Regions, eds. M. Peimbert and J. Jugaku (Dordrecht: Reidel) p. 213.

Snell, R.L., and Edwards, S. 1981, Ap. J., 251, 103.

Snell, R.L., Loren, R.B., and Plambeck, R.L. 1980, Ap. J. (Letters), 239, L17.

Snell, R L., Bally, J., Strom, S.E., and Strom, K.M. 1985, Ap. J., 290, 587.

Snell, R.L., and Bally, J. 1986, Ap. J., 303, 683 .

Snell, R.L., Huang, Y.-L., Dickman, R.L., and Claussen, M.J. 1988, Ap. J., 325, 853.

Snell, R.L., Dickman, R.L., and Huang, Y.-L. 1989, in preparation.

Strom, K.M., Strom, S.E., Wolff, S.C., Morgan, J., and Wenz, M. 1986, Ap. J. Suppl., 62, 39.

Terebey, S., Vogel, S.N., and Myers, P.C. 1989, $A p$. J., 340, 472.

Uchida, Y., Kaifu, N., Shibata, K., Hayashi, S.S., and Hasagawa, T. 1987, in IAU Symposium 115, Star Forming Regions, eds. M. Peimbert and J. Juku (Dordrecht: Reidel), p. 287. 


\section{Discussion:}

FRANCO: I have a question and a comment. There is a similar analysis recently made by Russ Levreault, but he reached the opposite conclusion (that outflows are not suitable to support the cloud). Can you clarify the reasons for this apparent contradiction? The comment, on the other hand, is that the results presented by G. Tenorio-Tagle and myself show that both photoionization and photodissociation fronts are capable of creating high-velocity neutral outflows. This mechanism can provide the momentum required to drive the molecular outflows.

SNELL: How important molecular outflows are in supporting clouds is certainly an open question. They must provide some support, the question is if they are the dominant source of support. The best way to address this question is through unbiased surveys of molecular clouds for outflows to provide a complete census. Russ Levreault has not done such a survey and thus does not have a coupled census of outflows in the clouds he studied. Complete surveys of clouds coupled with accurate measurements of their energy and momentum are necessary to answer this question.

SOLOMON: The outflow lifetime is critical to the question of cloud support. How accuratly is the lifetime determined and what are the assumptions?

SNELL: The outflow lifetimes are very poorly known, since they require a detailed knowledge of the structure of kinematics of the molecular gas as well as the inclination angle of the outflow. Lifetimes are usually computed from the projected size of the outflow divided by the radial velocity (often a mass weighted radial velocity is usual). It is important to find more accurate means to derive lifetimes.

LARSON: You mentioned the possible role of outflows in generating turbulence that helps to support molecular clouds. Is there evidence that clouds containing outflows have been stirred up or otherwise influenced by the outflows in regions that are not obviously part of an outflow?

SNELL: I believe there is such evidence, but it is difficult to prove it is due to molecular outflows. In the Taurus dark clouds there are several old outflows that can still be connected to their stellar source; these outflows are decelerating and have relatively large masses and low velocities. The spectra of these old outflows have, instead of high velocity wings, two components; one componet due to the ambient gas and a second one due to the swept-up massive shell. Much of the multiple velocity structure seen in Taurus and other clouds may well be due to these decelerating shells. 